\title{
Effects of porous trailing edge on aerodynamic noise characteristics
}

\author{
Minghui Zhang ${ }^{1}$ Tze Pei Chong ${ }^{2}$ \\ 1. Department of Mechanical and Electronic Engineering, Shandong University of Science \\ and Technology, Qingdao, 266590, China
2. Department of Mechanical and Aerospace Engineering, Brunel University London, Uxbridge, UB8 3PH, United Kingdom

\begin{abstract}
The objective of this work is to investigate the effect of the porous trailing edge on the aeroacoustics performance of the NACA 65(12)-10 aerofoil. The motivation behind this study is to investigate the effect of the porous parameters to explore the noise control concepts. Experimental testing in an aeroacoustics open jet wind tunnel was performed at chord-based Reynolds numbers between 0.2 and 0.6 million, and effective angles of attack at \pm 1.7 degree, including at 0 degrees. The porous trailing edge at porosity $30 \%$ with different holes diameters and the length of these porous trailing edges are used in the acoustic experiments. The study reveals that the level of the reduction of the broadband noise becomes larger as the diameter of the holes decreases and the length of the porous trailing edge increases at lower Reynolds numbers. Bluntness-induced tone noise is produced at high Reynolds number. Meanwhile, the porous trailing edge can suppress the laminar instability noise at the middle and low frequency regions.
\end{abstract}

Keywords: airfoil self-noise, porous trailing edge, pole distribution, parameters effect, tonal noise reduction

\section{Introduction}

In recent years, the subject of aerodynamic noise and its control have attracted much interest from the aeroacoustics research community [1]. A number of methods and devices (active and passive) have been investigated with the aim to reduce the aerodynamic noise. Among of them, is the bio-inspired technique to control aerodynamic noise which has a great potential. For example, the owl's wings have the well-known ability of silent flight [2] due to three distinctive and unique characteristics, namely, the serrated feathers on the leading edges, the fringes formed at the trailing edges, and the soft downy coating on the surface of wings and legs. In some senses, the third feature can be engineered by producing porous materials with different open and mutually inter-connected pores.

In an experimental study, Angland et al. [3] found that porous media can reduce the noise generation from flap-side edges. Geyer et al. [4-6] manufactured several SD7003 aerofoils with different porous materials. It was found that the level and characteristic of noise reduction by their treated aerofoils depend on the flow resistivity, and to some extents, the porosity. When different permeable materials were implemented at the trailing edge of a DLRF16 aerofoil, Herr et al. [7] observed that most of the noise reductions occur at a lower frequency. In particular, trailing edge with a higher permeability level could also affect the frequency range for the trailing edge noise reduction [8]. Porous trailing edges with sub-millimeter pore sizes, such as the open-cell $\mathrm{Ni}-\mathrm{Cr}$-Al foams used by Hedayati et al. [9], have been found to produce encouraging results for the reduction of broadband trailing edge noise.

In numerical analyses, Weidenfeld et al. [10] investigated the effect of structure permeability of a thin aerofoil subjected to low-Mach number and high-Reynolds number flow at flapping-flight and unsteady flow conditions. In their numerical simulation, aerofoil porosity was modeled using Darcy's law, and governed by a prescribed distribution of surface intrinsic permeability. Divya et al. [11] performed a parametric analysis for an aerofoil with a poro-elastic carpet on the suction side. It shows that such a coating is able to affect the topology of the flow in the proximity of the rear of the aerofoil, by adapting spontaneously to the separated flow. Shan $e t$ al. [12] investigated the effect of porous surface and expanded their study to the aerodynamic characteristics. Sarradj et al. [13] applied symbolic regression of large data set to develop the empirical models capable of describing the noise generation at porous aerofoils. In a numerical study of a new porous wall model, Nambu et al. [14] found that a porous wall is effective for reducing blockage, which then affects the Mach number around the aerofoil. For aerofoil fitted with morphing trailing edges, a coupled structure/fluid/noise model implemented by Qing et al. [15] demonstrated that an appropriate morphing profile tailoring can improve the aerodynamic and aeroacoustics performance of an aerofoil. Seong et al. $[16,17]$ analysed the impact of porous material with variable properties on trailing edge noise by a high resolution large-eddy simulation/computational 
aeroacoustics (LES/CAA) approach. It is shown that the viscous dissipation in the porous structures directly influences the acoustic attenuation through the reduction of the turbulent eddies correlation length under the backdrop of flow acceleration near the trailing edge. Zhou et al. [18] developed a discrete adjoint framework with porous media on the basis of algorithmic differentiation for trailing edge noise minimisation. They predicted a maximum noise reduction of $12 \mathrm{~dB}$ from a flat plate trailing edge. Recently, Miller and Pager [19] developed a semi-empirical mathematical model depending on the local flow field statistics to predict and analyse the acoustic radiation from turbulent boundary layers over porous media. It is found that noise is amplified or reduced in a nonintuitive way with the introduction of porosity, variation of frequency, and increase of Mach number.

Recently, the rapid advances of the additive manufacturing technique, such as the 3D-printing, could provide an alternative for the manufacture of permeable trailing edge inserts. Jiang et al. [20] produced porous rotor blades by $3 \mathrm{D}$ printing technique, where aerodynamic noise reduction was observed. Chen [21] designed a trailing edge with perforation treatment in order to reduce tonal noise of a contra-rotating fan. Carpio et al. [22] measured the far-field noise radiated with porous trailing edge inserts. It is observed that the 3D-printed inserts must be at least 3 times as permeable as the metal foam ones in order to obtain similar broadband noise attenuation levels. Jothi [23] implemented line distribution of $3 \mathrm{~mm}$ diameter holes adjacent to the trailing edge in their aeroacoustics investigation. The results indicate that the treated aerofoil is effective in the reduction of the lower frequency noise compared to that of the reference aerofoil.

In all previous studies, the researchers focused on the relation between different permeable materials, the pore characteristics, different flow resistivities and the degree of noise reduction with the fixed length of the porous trailing edge. In addition, little research has been done on the effects of porous trailing edge on laminar instability tonal noise. As part of efforts to fill the knowledge gap, one baseline trailing edge and 18 porous trailing edge structure with porosity $30 \%$, including different diameter of the hole, the length of the porous trailing edge, were investigated for their noise performance. This paper focused on the following areas: (1) how does the hole diameter affect the trailing edge noise; (2) how does the length of the porous trailing edge affect the trailing edge noise; (3) how does the change of angle of attack affect the trailing edge noise; and (4) how does the prorous trailing edge affect the laminar instability tonal noise.

The remainder of the paper is organized as follows: Section 2 briefly reviews the experimental setup in detail including wind tunnel facilities and acoustic measurement and the airfoil models. In Sections 3, the results of the different experiments are presented and discussed. Finally, the findings of the present study are concluded in Section 4.

\section{Experimental set-up}

\subsection{Wind Tunnel Facilities and Acoustic Measurement}

Noise measurements were conducted in an aeroacoustic open jet wind tunnel at Brunel University London. The open jet wind tunnel is situated in a $4 \mathrm{~m} \times 5 \mathrm{~m} \times 3.4 \mathrm{~m}$ anechoic chamber to facilitate free field measurement of the airfoil self-noise. As shown in Fig. 1, the nozzle exit is rectangular with dimensions of $100 \mathrm{~mm}$ (height) $\times 300 \mathrm{~mm}$ (width). This wind tunnel can achieve a turbulence intensity of between $0.1 \%$ to $0.2 \%$ and a maximum jet velocity of about $80 \mathrm{~m} / \mathrm{s}$. The airfoil model is held by side plates, which is used to adjust the angle of attack and the position of the airfoil regarding to the nozzle of the wind tunnel. There are eight condenser microphones at polar angles of $\theta\left(\theta=50^{\circ}, 60^{\circ}, 70^{\circ}, 80^{\circ}, 90^{\circ}, 100^{\circ}, 110^{\circ}, 120^{\circ}\right)$ at a distance of $1 \mathrm{~m}$ from the trailing edge. The wind tunnel self-noise is measured between 20 and $60 \mathrm{~m} / \mathrm{s}$ (interval $10 \mathrm{~m} / \mathrm{s}$ ), corresponding to Reynolds numbers $2 \times 10^{5}$ and $6 \times 10^{5}$, respectively, based on the chord.



Fig. 1. Picture showing the Brunel aeroacoustic wind tunnel 


\subsection{Airfoil Models}

The current paper aims at investigating the aerodynamic noise characteristics with the porous trailing edge to include the parametric effect of porous trailing-edge modification. The airfoil is a NACA 65(12)-10, with nominal chord length $c=150 \mathrm{~mm}$ and nominal span length $L=300 \mathrm{~mm}$. The model depicted in Fig. 2(a), are 3Dprinted with Viper Si2 SLA system. It contains exchangeable trailing edge section for both the porous and baseline trailing edges. The porous trailing edge covers the last from $0-20 \%(0-30 \mathrm{~mm})$ of the chord (Fig. 2 (b)), where $h_{\mathrm{c}}$ is the length of the trailing edge.

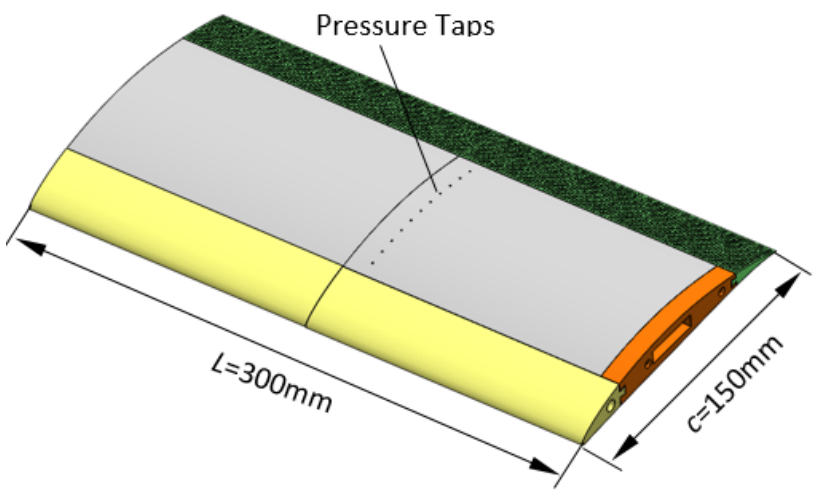

(a) Perspectiveview

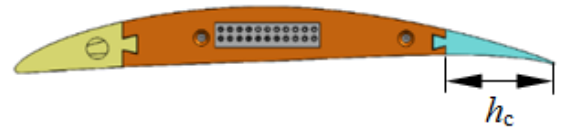

(b) Sideview

Fig. 2 Sketch of the NACA 65(12)-10 airfoil with 3D-printed

For the investigation of noise produced by the porous trailing edge with variations hole diameters and different length $h_{\mathrm{c}}$, eighteen types of porous trailing edge were designed and tested. The experimental results from the references $[7,23]$ show that the reduction of the broadband noise is more significant when the porosity increases. Taking into account of the minimal structural integrity needed for a porous trailing edge, as well as the limitation of accuracy for the 3D printer, we choose the porosity to be $30 \%$. For each porous trailing edge, a solid trailing edge was tested at the same flow conditions to allow comparisons of the noise data. The porous trailing edges were split into two sets and the parameters of the trailing edge were listed in Tables 1 and 2 . The pole distribution is based on the repetition of the squared pattern depicted in Fig. 3, where the diameter of holes is $d, t$ is the distance between the two holes along stream-wise, $h$ is the distance along the spanwise direction and $t_{\mathrm{h}}$ is the height of the first hole. The porosity of the trailing edge $\sigma$ is defined as:

$$
\sigma=\frac{V_{\text {hole }}}{V_{\text {total }}}
$$

where $V_{\text {hole }}$ and $V_{\text {total }}$ are the cummulative volume of the holes and the total volume of the trailing edge base they occupy, respectively.

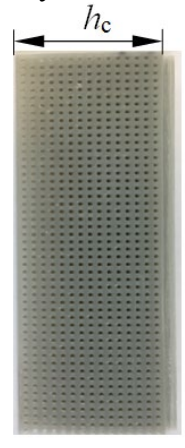

(a) Insert trailing edge.

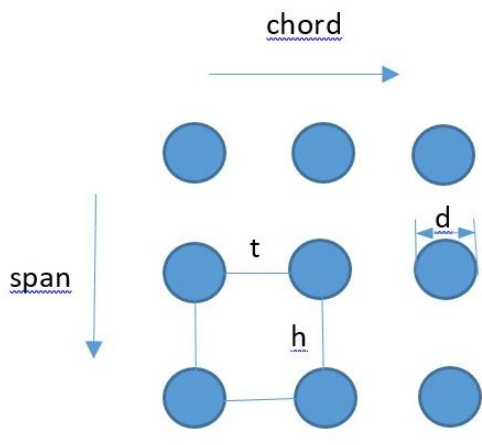

(b) Sketch of the hole pattern

Fig. 3 The hole pattern of the trailing edge

In the first set of test, in order to investigate the noise of the trailing edge with variations diameter of the hole, one baseline trailing edge and 3 porous trailing edge structures with the length $20 \%$ chord were used in the acoustic experiments. Tab. 1 summarizes the geometrical parameters of the trailing edge devices investigated in this study. To make it easier to differentiate the trailing edge devices, they are named according to the values of hole diameter $d$ and porisity $\sigma$. For example, a porosity trailing edge with a porosity $\sigma=30 \%$ and hole diameter $d=1 \mathrm{~mm}$ will be named as $30-1 \mathrm{~mm}$. Note that Rw is the total numer of row for the porous holes for a particular $\sigma$ and $d$ combination along the chordwise direction. 
Tab. 1 the geometrical parameters for the first set of test

\begin{tabular}{|l|c|c|c|c|c|c|c|c|}
\hline Symbols & $\begin{array}{c}\text { Types of } \\
\text { TE }\end{array}$ & $d(\mathrm{~mm})$ & $t(\mathrm{~mm})$ & $h(\mathrm{~mm})$ & $\mathrm{Rw}$ & $h_{\mathrm{c}}(\mathrm{mm})$ & $t_{\mathrm{h}}(\mathrm{mm})$ & $\sigma$ \\
\hline baseline & Baseline & 0 & 0 & 0 & 0 & 30 & 7.07 & 0 \\
\hline $30-1 \mathrm{~mm}$ & Poro TE & 1 & 1.5 & 1.6 & 19 & 30 & 6.89 & $30.402 \%$ \\
\hline $30-2 \mathrm{~mm}$ & Poro TE & 2 & 3.2 & 3.1 & 9 & 30 & 6.63 & $29.74 \%$ \\
\hline $30-3 \mathrm{~mm}$ & Poro TE & 3 & 4.8 & 4.65 & 6 & 30 & 6.39 & $30.02 \%$ \\
\hline
\end{tabular}

In the second set of test, in order to investigate the noise of the porous trailing edge with variations of the length, 15 porous trailing edges were designed. The porous trailing edge covers between 0 and $20 \%(0-30 \mathrm{~mm})$ of the chord, counted from the trailing edge. The rows of the hole Rw varied from 1 to 15 , hole diameter $d$ $=1 \mathrm{~mm}$ and porosity $\sigma=30 \%$. To make it easier to differentiate the trailing edge devices, they are named according to the rows of the hole. For example, a porosity trailing edge that has 8 rows in the trailing edge will be named as Row-8. Tab. 2 summarizes the geometrical parameters of the trailing edge devices investigated in this study.

Note that, except in Section 3.4 when the focus is on the laminar instability tonal noise, boundary layer tripping was applied near the leading edge at both the suction and pressure side of the airfoil to trigger a bypass transition so that fully developed turbulent boundary layer is produced at the trailing edge.

Tab. 2 the geometrical parameters for the second set of test

\begin{tabular}{|c|c|c|c|c|c|c|c|c|}
\hline Symbols & Types of TE & $d(\mathrm{~mm})$ & $t(\mathrm{~mm})$ & $h(\mathrm{~mm})$ & $t_{h}(\mathrm{~mm})$ & $\mathrm{Rw}$ & $h_{\mathrm{c}}(\mathrm{mm})$ & $\sigma$ \\
\hline Row-15 & Poro TE & 1 & 1.5 & 1.6 & 5.3 & 15 & 23.5 & $30.402 \%$ \\
\hline Row-14 & Poro TE & 1 & 1.5 & 1.6 & 4.94 & 14 & 22 & $30.402 \%$ \\
\hline Row-13 & Poro TE & 1 & 1.5 & 1.6 & 4.58 & 13 & 20.5 & $30.402 \%$ \\
\hline Row-12 & Poro TE & 1 & 1.5 & 1.6 & 4.23 & 12 & 19 & $30.402 \%$ \\
\hline Row-11 & Poro TE & 1 & 1.5 & 1.6 & 3.87 & 11 & 17.5 & $30.402 \%$ \\
\hline Row-10 & Poro TE & 1 & 1.5 & 1.6 & 3.52 & 10 & 16 & $30.402 \%$ \\
\hline Row-9 & Poro TE & 1 & 1.5 & 1.6 & 3.18 & 9 & 14.5 & $30.402 \%$ \\
\hline Row-8 & Poro TE & 1 & 1.5 & 1.6 & 2.84 & 8 & 13 & $30.402 \%$ \\
\hline Row-7 & Poro TE & 1 & 1.5 & 1.6 & 2.5 & 7 & 11.5 & $30.402 \%$ \\
\hline Row-6 & Poro TE & 1 & 1.5 & 1.6 & 2.17 & 6 & 10 & $30.402 \%$ \\
\hline Row-5 & Poro TE & 1 & 1.5 & 1.6 & 1.85 & 5 & 8.5 & $30.402 \%$ \\
\hline Row-4 & Poro TE & 1 & 1.5 & 1.6 & 1.54 & 4 & 7 & $30.402 \%$ \\
\hline Row-3 & Poro TE & 1 & 1.5 & 1.6 & 1.25 & 3 & 5.5 & $30.402 \%$ \\
\hline Row-2 & Poro TE & 1 & 1.5 & 1.6 & 0.98 & 2 & 4 & $30.402 \%$ \\
\hline Row-1 & Poro TE & 1 & 1.5 & 1.6 & 0.75 & 1 & 2.5 & $30.402 \%$ \\
\hline
\end{tabular}

\section{Results and discussion}

This section will present the experimental results pertaining for the broadband noise reduction by the porous leading edges. The relatively large data set from the measurements cannot be fully presented here. Thus, only selected results are given that allow the assesssment of the influence of porous trailing edge parameters on the sound generation of airfoils.

The noise radiation is investigated in terms of Sound Power Level spectrum. Assuming a cylindrical radiation, since the radiation from an airfoil resembles more closely a line source than a point source, the sound power level per unit span PWL obtained between radiation angles of $50^{\circ}$ and $120^{\circ}$ is given in Equation (2):

$$
\left\{\begin{array}{c}
W(f)=\frac{2 \pi r \sum_{i} \phi_{p p}\left(f, \theta_{i}\right) \Delta \theta}{\rho c_{0}} \\
P W L(f)=10 \log _{10}\left(W(f) / W_{0}\right)
\end{array} \quad i=1,2, \cdots N, 50^{\circ}<\theta<120^{\circ}\right.
$$

where $W(f)$ is the sound power integrated between the radiation angles $50^{\circ}$ to $120^{\circ}, W_{0}=10^{-12} \mathrm{~W} / \mathrm{Hz}$, $\phi_{p p}\left(f, \theta_{i}\right)$ is the pressure spectrum density measured at microphone $i, N$ is the number of microphones, $r$ is the distance between the airfoil trailing edge and the observer, $\Delta \theta=10^{\circ} \times \pi / 180$ is the angle between adjacent microphones and $c_{0}$ is the speed of sound.

The reduction in the sound power levels $(\triangle P W L)$ is expressed as the difference between the sound power level of the porous airfoil and the sound power level of the baseline airfoil, as shown in the Equation (3). 


$$
\Delta P W L=P W L^{\text {porous }}-P W L^{\text {baseline }}
$$

\subsection{Effect of hole diameter on the noise spectra}

This section investigates the variation of the sound power level radiated from the porous trailing edge with different $d$, as listed in Table 1. Trailing edge noises are presented under flow speed $U_{0}=20-60 \mathrm{~m} / \mathrm{s}$ for the porosity $\sigma=30 \%$, hole diameter $d=1 \mathrm{~mm}, 2 \mathrm{~mm}, 3 \mathrm{~mm}$ inserts. Acoustic spectra for the baseline case is also included for comparison.

The sound power level spectra at the velocity $U_{0}=20 \mathrm{~m} / \mathrm{s}$ is shown in Fig. 4(a). For the $d=2 \mathrm{~mm}$ insert a similar performance as the baseline case is evident up to $3 \mathrm{kHz}$, while the $d=1 \mathrm{~mm}$ insert produces lower sound power level. The $d=3 \mathrm{~mm}$ insert produces sound power level higher than the baseline trailing edge over almost the entire frequency range. The difference between far-field noise for the porous treatments with respect to the baseline insert $\triangle P W L$ is shown in Fig. 4(b). It shows noise reduction for the hole diameter $d=1 \mathrm{~mm}$ at the frequency between $235 \mathrm{~Hz}$ and $3680 \mathrm{~Hz}$, while for higher frequencies, this particular porous insert will produce higher noise level. For the $d=2$ and $3 \mathrm{~mm}$ cases, noise increase is prominent.

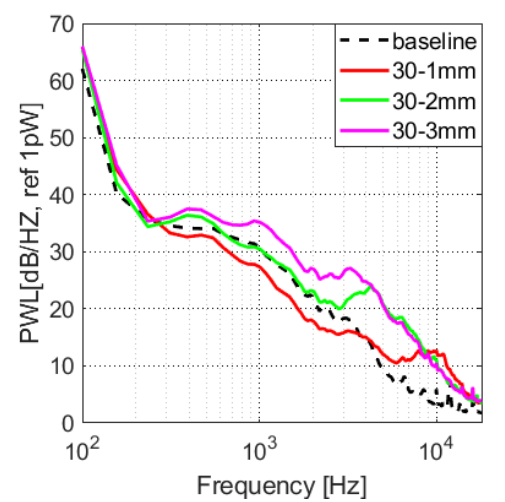

(a) $P W L$ at the different hole diameter

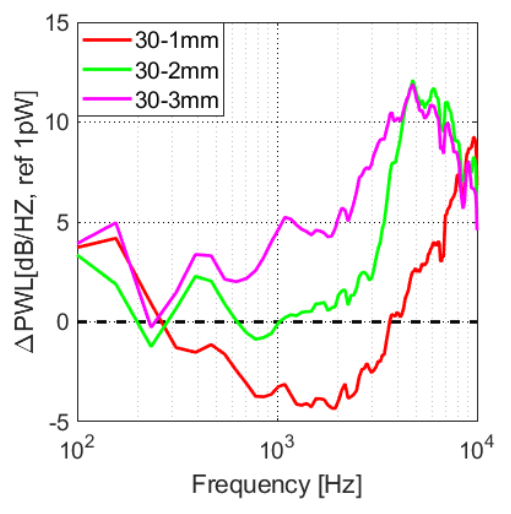

(b) The reduction in the sound power levels

Fig. 4 A comparison between baseline edge and porous edges, $U_{0}=20 \mathrm{~m} / \mathrm{s}$

The sound power level spectra at the velocity $U_{0}=60 \mathrm{~m} / \mathrm{s}$ are presented in Fig. 5. Generally, the noise amplitude is increased as increasing the hole diameter in the mid-frequency range. In Fig. 5(a), it is noticed that there is no noise-reduction effect for the three hole diameter configurations. Furthermore, large tonal peaks are very prominent when the hole diameter is at $d=1 \mathrm{~mm}$.

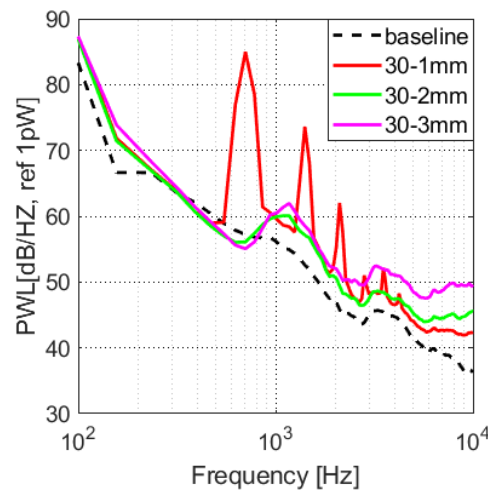

(a) $P W L$ at the different hole diameter

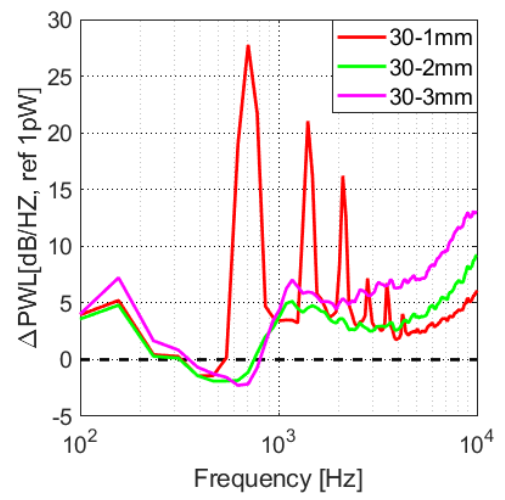

(b) The reduction in the sound power levels

Fig. 5 A comparison between baseline straight edge and porous edges, $U_{0}=60 \mathrm{~m} / \mathrm{s}$

In order to investigate the changes in sound power radiation with varying mean flow velocity, Figure 6 shows the typical behaviour of the sound power level reduction $\triangle P W L$, for 3 porous trailing edge structure at $0^{\circ}$ angle of attack, as a function of frequency and mean flow velocity $U_{0}$. Note that the limits of $\triangle P W L= \pm 5 \mathrm{~dB}$ in Figure 6 are set to emphasize the behaviour of the transition frequency between sound power reduction and sound power increase, and they do not refer to the maximum and minimum changes in sound power level. Noise increase represented by the streak-like positive $\triangle P W L$ in the contour can be reduced by increasing the diameter 
of the hole. For the broadband noise, decreasing the hole diameter improves the level of broadband noise reduction, with the efficiency more prominent at low velocity.

To demonstrate the PWL spectra, Figure 7 shows the sound power level with varying mean flow velocity $U_{0}=30$ and $50 \mathrm{~m} / \mathrm{s}$. For the hole diameter $d=1 \mathrm{~mm}$, the numerous tonal peak occur when the mean flow velocity reaches $30 \mathrm{~m} / \mathrm{s}$, and the tonal peak become larger in $P W L$ with increasing the flow velocity. For the hole diameter $d=2 \mathrm{~mm}, 3 \mathrm{~mm}$, no tonal peaks are observed throughout the velocity range.

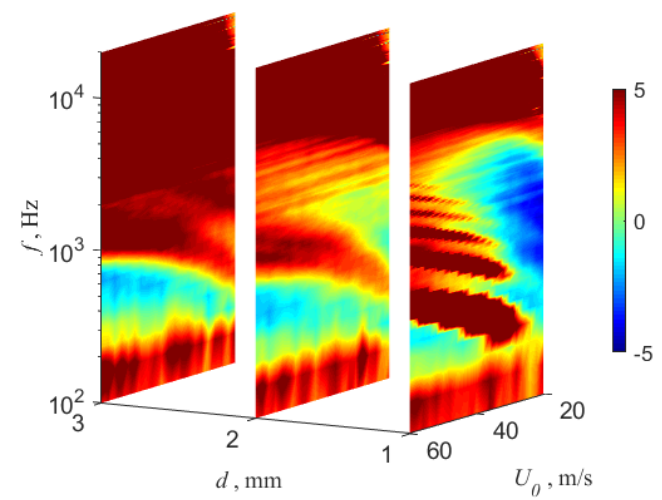

Fig. 6 Contour maps of the $\triangle P W L$

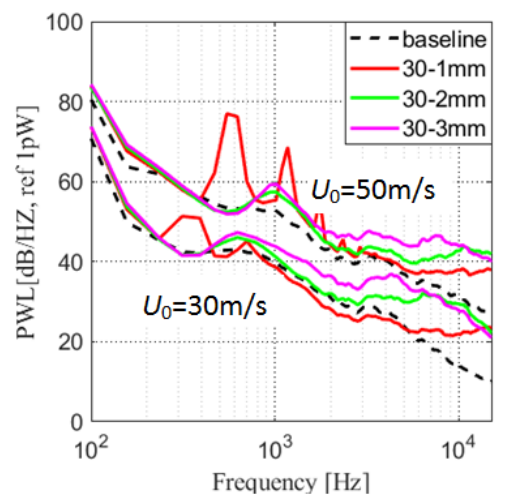

Fig. 7 Comparison of the $P W L$ at different speeds

At $d=1 \mathrm{~mm}$, the distance between the two holes along the spanwise direction is only $1.6 \mathrm{~mm}$, which is close to having a two-dimensional blunt edge. However, for cases $d=2 \mathrm{~mm}$ and $3 \mathrm{~mm}$, the distance between the two holes along the spanwise is relatively large $(3.1 \mathrm{~mm}$ and $4.65 \mathrm{~mm}$, respectively). The origin of the tonal peak observed in Fig. 7 for the low $d$ case is therefore likely to be related to those two-dimensional bluntness-induced vortex shedding, i.e. presence of large value of $t_{h}$ and small value of $t$ and $h$ (see Tables 1 and 2), although no experimental evidence is available at this stage.

\subsection{Effect of the hole rows on the noise spectra with speed}

In order to to further investigate the noise of the porous trailing edge with variations of the length, 15 porous trailing edge structure was designed, with the hole diameter $d=1 \mathrm{~mm}$, the porosity $\sigma=30 \%$. The parameters are listed in Table 2. Acoustic measurements have been conducted at $0^{\circ}$ angle of attack and the mean flow velocity $U_{0}=20-60 \mathrm{~m} / \mathrm{s}$.

Figure 8 presents the typical behaviour of the sound power level reduction $\triangle P W L$, for 15 porous trailing edge structure, at $U_{0}=20 \mathrm{~m} / \mathrm{s}, U_{0}=30 \mathrm{~m} / \mathrm{s}$ and $U_{0}=40 \mathrm{~m} / \mathrm{s}$. The show that broadband noise reduction is achieved at $U_{0}=20 \mathrm{~m} / \mathrm{s}$ for all the cases, with the level of noise reduction increases as the number of hole row increases. At $U_{0} \geq 30 \mathrm{~m} / \mathrm{s}$, tonal peaks begin to occur at low frequency when the hole row is larger than 10 . When the number of row is less than or equal to 10, the tendency to produce the extraneous tonal peak becomes larger at high speed and also moves to a higher frequency.

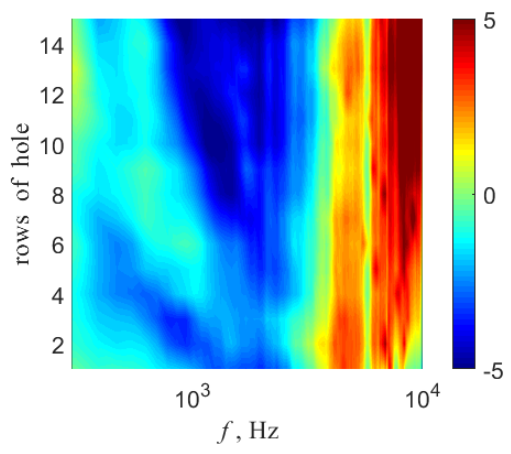

(a) $U_{0}=20 \mathrm{~m} / \mathrm{s}$

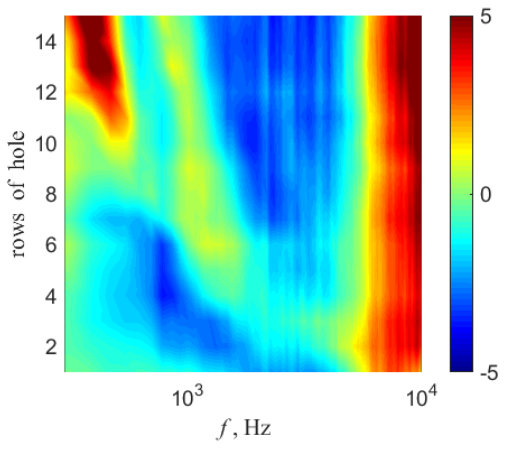

(b) $U_{0}=30 \mathrm{~m} / \mathrm{s}$

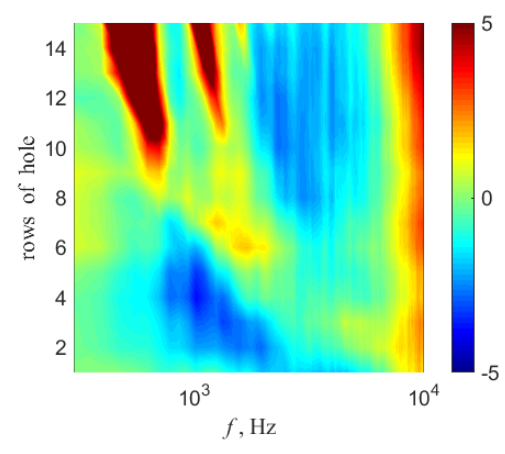

(c) $U_{0}=40 \mathrm{~m} / \mathrm{s}$

Fig. 8 Contour maps of the $\triangle P W L$ with different length of the porous trailing edge

The $P W L$ of the porous trailing edge with different Row number against speeds is presented in Fig. 9. The figure reveals that tne tonal peak becomes more prominent as both the row number and speed increase. Again, a larger value of row number denotes a larger value of $t_{h}$, which represents a hint that the origin of these tones is likely to be the bluntness-induced vortex shedding. 


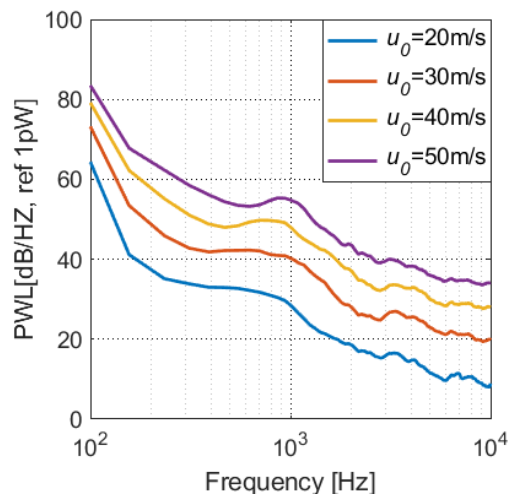

(a) Row-8

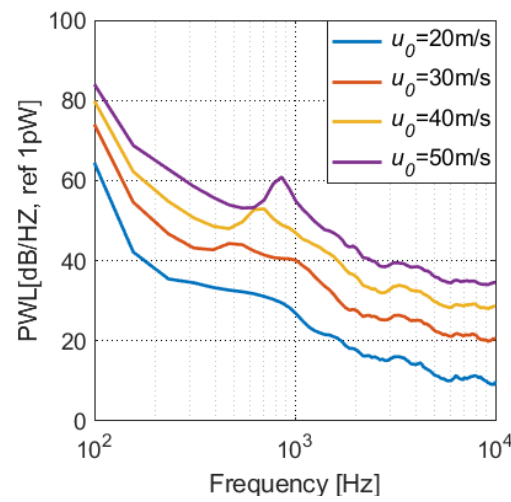

(c) Row-10

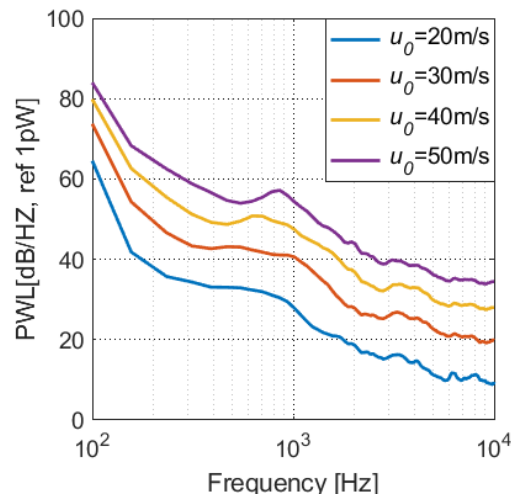

(b) Row-9

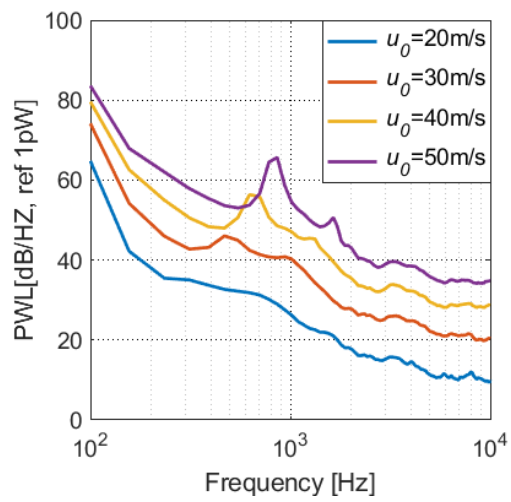

(d) Row-11

Fig. 9 the $P W L$ of the porous trailing edge of with different speeds

In order to study the mechanism of occurrence vortex shedding occurence, boundary layer thickness of the baseline at different position of the trailing edge at $U_{0}=50 \mathrm{~m} / \mathrm{s}$ were predicted in numerical simulation. Because the boundary layer displacement thicknesses between the upper and lower surfaces of the airfoil are different, the mean value between them $\delta^{*}$ is instead taken:

$$
\delta^{*}=\left(\delta^{*}{ }_{1}+\delta^{*}{ }_{2}\right) / 2
$$

where $\delta^{*}$ and $\delta^{*}{ }_{2}$ are the turbulent boundary layer displacement thickness at the suction and pressure sides, respectively (see Fig. 10(a)). The values in Fig. 10a were numerically calculated by the commercial software FLUENT based on turbulence model ( $\kappa-\omega$ SST model) of the flow field. In order to evaluate turbulence model and the calculated results, comparison of the thickness of boundary layer $\left(\mathrm{U}_{0}=24 \mathrm{~m} / \mathrm{s}\right)$ for the baseline airfoil between the numerical and experimental results [25] is quite good. The same turbulence model and numerical procedure was used to calculate the thickness of boundary layer for the case when $\mathrm{U}_{0}=50 \mathrm{~m} / \mathrm{s}$.

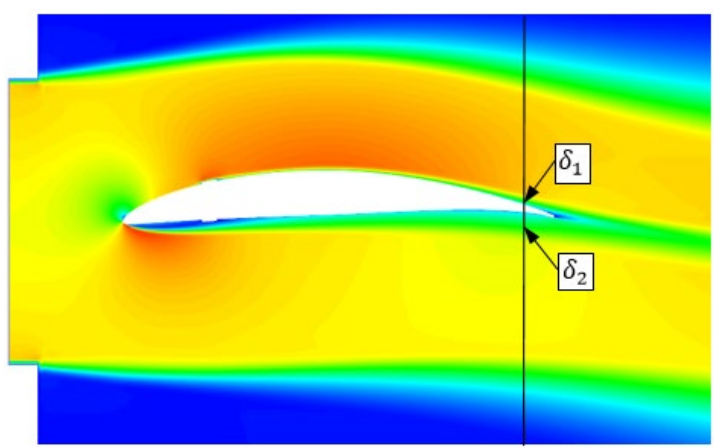

Fig. 10 Streamwise velocity contour and the position for obtain boundary layer thickness

After analysing all the data set, the presence of the vortex shedding is found to correlate to the local turbulent boundary layer displacemnt thickness $\delta^{*}$ and $t_{h}$. The condition for tonal noise to occur should satisfy the following function: 


$$
\frac{t_{h}}{\delta^{*}}<2
$$

Note that the value above, which is related to a porous trailing edge, is understandably to be larger than the reported value of 0.3 [26] for a completely two-dimensional blunt trailing edge.

\subsection{Noise reduction at different angles of attack}

This section investigates the variation of the sound power level radiated from the porous trailing edge with varying mean flow velocity at different geometrical attack angles from $-10^{\circ}$ to $10^{\circ}$. For the flow from the open-jet wind tunnel, which will deflect downwards, it is important to apply correction in order to determine the effective angle of attack $\alpha_{e}$ in free air. Equation (6) indicates that the geometrical angle of attack $\alpha_{g}$ and is corrected by the geometrical factor $\zeta$ to obtain the equivalent angle in free air $\alpha_{e}$ for an equivalent lift force, it is used in the current study to estimate the angle of attack in free air.

$$
\alpha_{e}=\frac{\alpha_{g}}{\zeta}, \quad\left\{\begin{array}{c}
\zeta=(1+2 \sigma)^{2}+\sqrt{12 \sigma} \\
\sigma=\frac{\pi^{2}}{48}\left(\frac{c}{H}\right)^{2}
\end{array}\right.
$$

where $c$ and $\mathrm{H}$ are the airfoil chord and the height of the jet, respectively, for a horizontally aligned airfoil (with $c=0.15 \mathrm{~m}, \mathrm{H}=0.1$ in this study). The actual tunnel angles of attack investigated for noise reduction are listed with their free air equivalent values in Table 3.

Table 3 Angle of attack correction due to the flow deviation by the airfoil

\begin{tabular}{|c|c|c|c|c|c|}
\hline$\alpha_{g}$ & -10 & -5 & 0 & 5 & 10 \\
\hline$\alpha_{e}$ & -1.65069 & -0.82534 & 0 & 0.825345 & 1.650689 \\
\hline
\end{tabular}

Figure 11 shows the typical behaviour of the sound power level reduction $\triangle P W L$ for the case hc $=30 \mathrm{~mm}$ when the hole diameter $\mathrm{d}=1 \mathrm{~mm}$, porosity $=30 \%$, the attack angle at $-10^{\circ},-5^{\circ}, 0^{\circ}, 5^{\circ}$ and $10^{\circ}$. Note that hc is the length of the porous coverage. It clearly shows that the tonal peaks become less prominent at low to negative angles of attack. In addition, the frequency range over which broadband noise reduction occurs is also larger at a more negtive angle of attack. When the attack angle is equal to $0^{\circ}$ or large than $0^{\circ}$, the effect of reducing noise is similar with the case at the attack angle of $0^{\circ}$. In addition, with the speed increasing, the tonal peaks become more prominent.

Figure 12 shows the color map of the sound power level reduction $\triangle P W L$ for the case hc $=7 \mathrm{~mm}$ when the hole diameter $\mathrm{d}=1 \mathrm{~mm}$, porosity $=30 \%$, the attack angle at $-10^{\circ},-5^{\circ}, 0^{\circ}, 5^{\circ}$ and $10^{\circ}$. It can be seen from the figure that the level of broadband noise reduction is not as good as that of the porous trailing edge presented in Fig. 11. The advantage of the porous trailing edge in Fig. 12 is that no tonal peaks are produced throughout the entire range of angle of atack and flow speed investigated here.

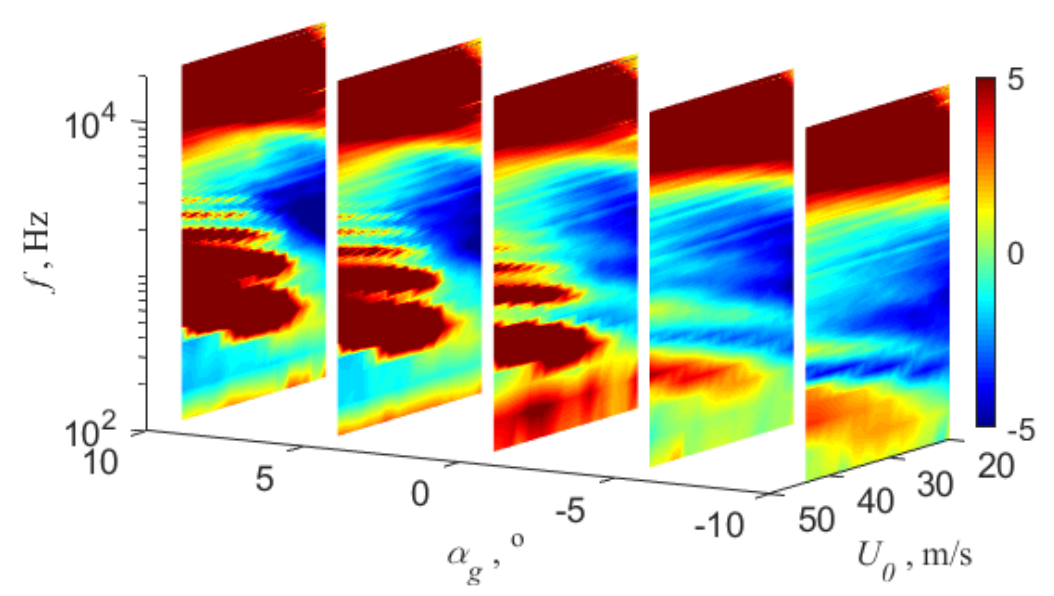

Fig. 11 the sound power level reduction for the case $h c=30 \mathrm{~mm}, d=1 \mathrm{~mm}$, porosity $=30 \%$ 


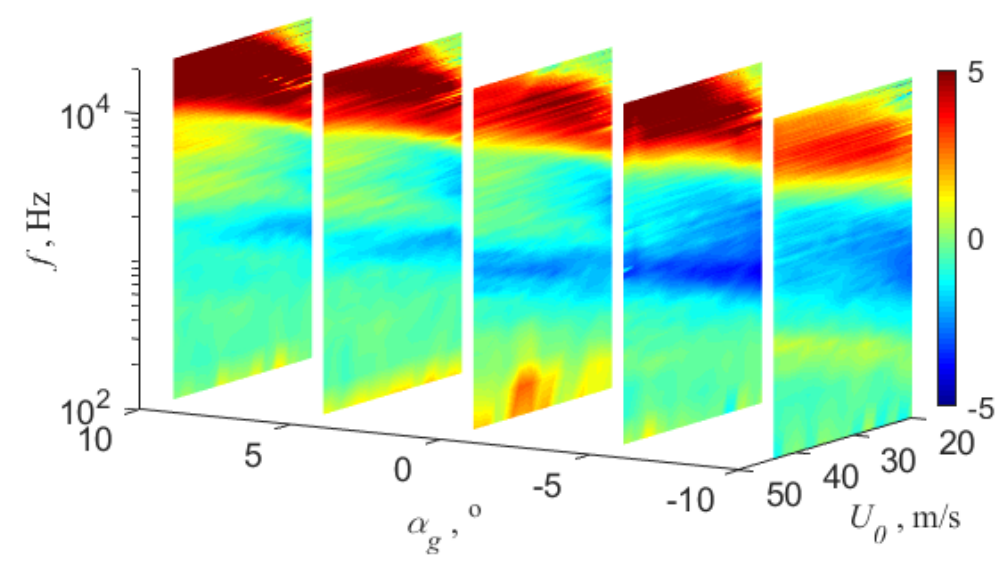

Fig. 12 the sound power level reduction for the case $\mathrm{hc}=7 \mathrm{~mm}, \mathrm{~d}=1 \mathrm{~mm}$, porosity $=30 \%$

The difference between far-field noise for the porous treatments with respect to the solid insert $\triangle P W L$ with the velocity $U_{0}=20 \mathrm{~m} / \mathrm{s}$, the attack angle at $-10^{\circ},-5^{\circ}, 0^{\circ}, 5^{\circ}$ and $10^{\circ}$ are shown in Fig. 13. It shows that, for $\mathrm{hc}=30 \mathrm{~mm}$ (denoted as $30-1 \mathrm{~mm}$ in the figure), noise reduction is achieved at mostly mid frequency region throughout the entire angles of attack investigated here. When hc is reduced to $7 \mathrm{~mm}$ (denoted as Row-4 in the figure), the frequency range underpinning the broadband noise reduction is similar, but the level of noise reduction is lower. However, the level of noise increase at high frequency is not as prominent. Table 4 shows the maximum noise reduction $\triangle P W L$ values with different angles of attack for the Row-4 and 30-1mm porous airfoil. When the angle of attack is less than or equal to $0^{\circ}$, the corresponding frequency to the maximum noise reduction amplitude is the same. When the angle of attack is greater than $0^{\circ}$, the corresponding frequency to the maximum noise reduction amplitude is higher with the length of the porous trailing edge increasing.

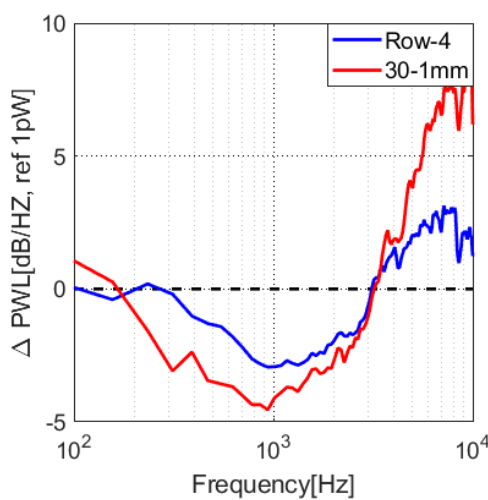

(a) $\alpha=-10^{\circ}$

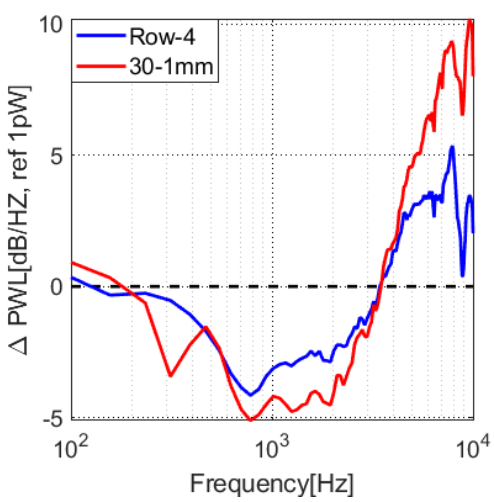

(b) $\alpha=-5^{\circ}$

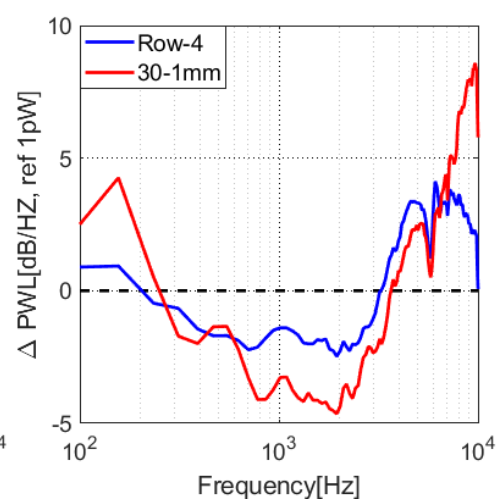

(c) $\alpha=0^{\circ}$

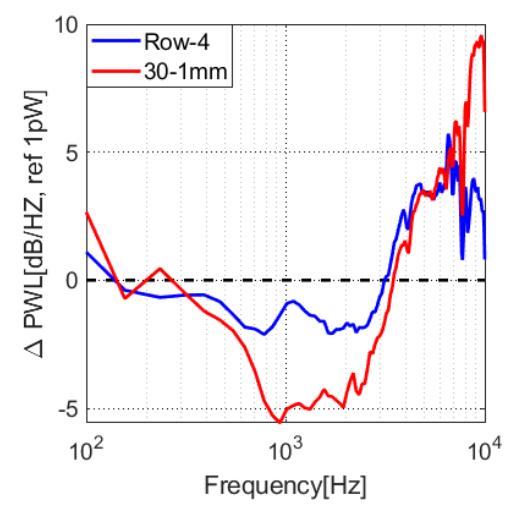

(c) $\alpha=5^{\circ}$

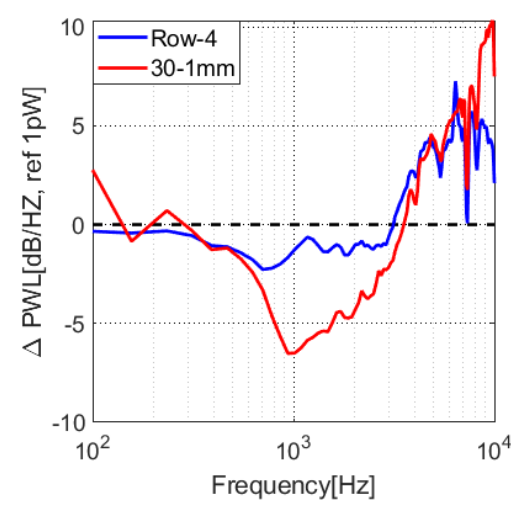

(d) $\alpha=10^{\circ}$

Fig. 13 the sound power level reduction for the case $\mathrm{d}=1 \mathrm{~mm}$, porosity $=30 \%, U_{0}=20 \mathrm{~m} / \mathrm{s}$ 
Table 4 Maximum reduction noise $\triangle P W L$ values

\begin{tabular}{|c|c|c|c|c|c|c|}
\hline & & $-10^{\circ}$ & $-5^{\circ}$ & $0^{\circ}$ & $5^{\circ}$ & $10^{\circ}$ \\
\hline \multirow{2}{*}{ Row-4 } & Frequency $[\mathrm{Hz}]$ & 937.5 & 781.3 & 1953 & 781.3 & 781.3 \\
\cline { 2 - 7 } & Max $\triangle P W L$ & -2.938 & -4.15 & -2.477 & -2.117 & -2.269 \\
\hline \multirow{2}{*}{$1 \mathrm{~mm}-30$} & Frequency $[\mathrm{Hz}]$ & 937.5 & 781.3 & 1953 & 937.5 & 937.5 \\
\cline { 2 - 7 } & Max $\triangle P W L$ & -4.545 & -5.112 & -4.64 & -5.572 & -6.513 \\
\hline
\end{tabular}

Fig. 14 shows the difference between far-field noise for the porous treatments with respect to the solid insert $\triangle P W L$ with the velocity $U_{0}=50 \mathrm{~m} / \mathrm{s}$, the attack angle at $-10^{\circ},-5^{\circ}, 0^{\circ}, 5^{\circ}$ and $10^{\circ}$. It shows that, for the airfoil with hc $=30 \mathrm{~mm}$ (denoted as $30-1 \mathrm{~mm}$ in the figure), there is no noise reduction effect with respective to the baseline and the strong tonal peaks appear when the speed reaches $50 \mathrm{~m} / \mathrm{s}$. As the angle of attack increases, the magnitude and number of tonal peaks increases. For the airfoil with hc=7 mm (denoted as Row -4 in the figure), no tonal peaks appear throughout the range of angle of attack investigated here. Generally, better noise performances for both porous trailing edges when they are at negative angle of attack.

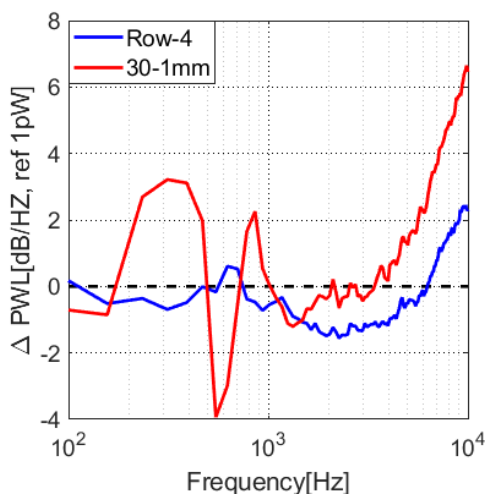

(a) $\alpha=-10^{\circ}$

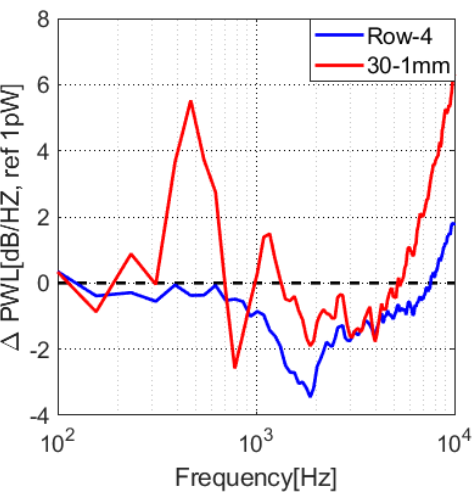

(b) $\alpha=-5^{\circ}$

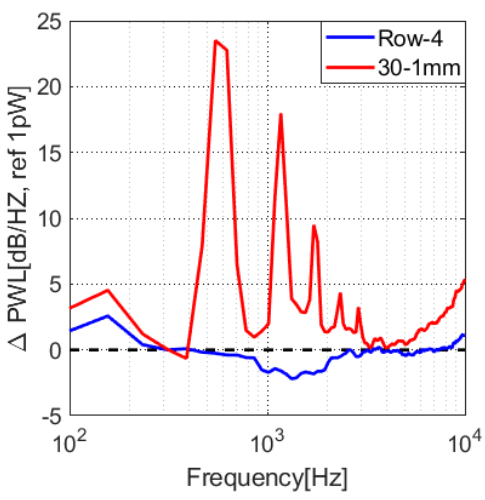

(c) $\alpha=0^{\circ}$

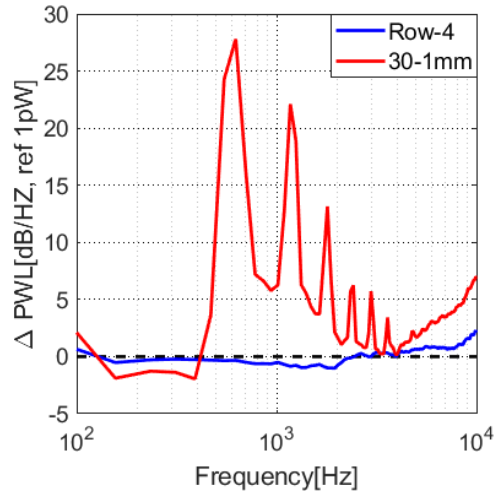

(c) $\alpha=5^{\circ}$

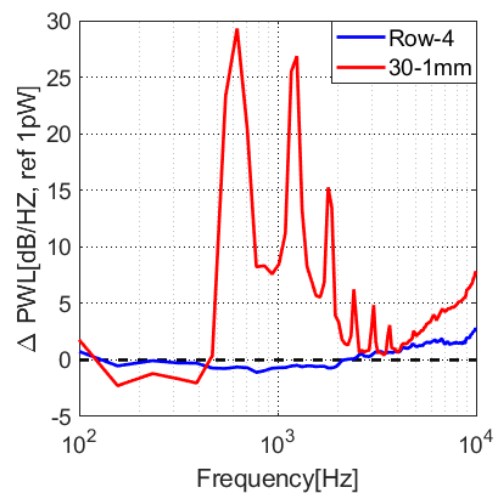

(d) $\alpha=10^{\circ}$

Fig. 14 the sound power level reduction for the case $\mathrm{d}=1 \mathrm{~mm}$, porosity $=30 \%, U_{0}=50 \mathrm{~m} / \mathrm{s}$

\subsection{Effect of porous trailing edge on the laminar instability tonal noise}

Acoustic measurements have been conducted at $0^{\circ}$ angle of attack, and the mean flow velocity $U_{0}=20-$ $60 \mathrm{~m} / \mathrm{s}$ when the airfoil is untripped in order to study the effects of porous trailing edge on the laminar instability tonal noise. Some $P W L$ spectra is presented in Fig 15 for the baseline, Row-4 (hc=7mm) and 30$1 \mathrm{~mm}(\mathrm{hc}=30 \mathrm{~mm})$ at $U_{0}=20-50 \mathrm{~m} / \mathrm{s}$. For the baseline airfoil, large tonal noise of boundary layer instability origin will be produced at low speeds and tonal noise moves to high frequency as speed increases. Fig 15 also shows that the porous trailing edge can suppress the laminar instability tonal noise, especially for the case where the 
length of the porous trailing edge is equal to $30 \mathrm{~mm}$, there is no tonal noise at the speed $U_{0}=20 \mathrm{~m} / \mathrm{s}$. The thickness of the first row hole near leading edge increased from $t_{h}=1.54 \mathrm{~mm}$ to $t_{h}=6.89 \mathrm{~mm}$ when the rows increased from 4 to 19. The level of bluntness-induced tonal peak increases with the thickness of blunt edge. Therefore, the long porous trailing edge $(30-1 \mathrm{~mm})$ will cause an additional bluntness-induced tonal peaks to jeopardize the overall performance especially at high high speed.

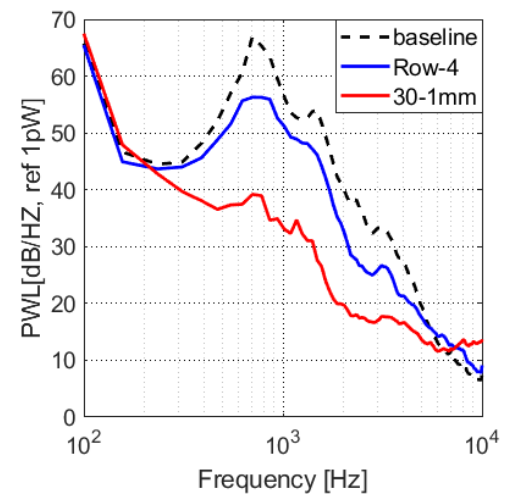

(a) $U_{0}=20 \mathrm{~m} / \mathrm{s}$

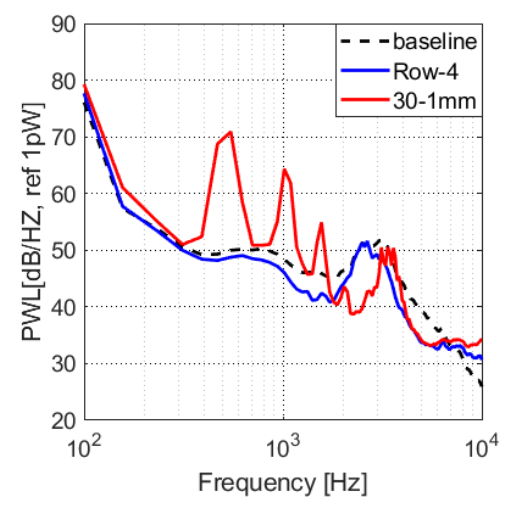

(c) $U_{0}=40 \mathrm{~m} / \mathrm{s}$



(b) $U_{0}=30 \mathrm{~m} / \mathrm{s}$

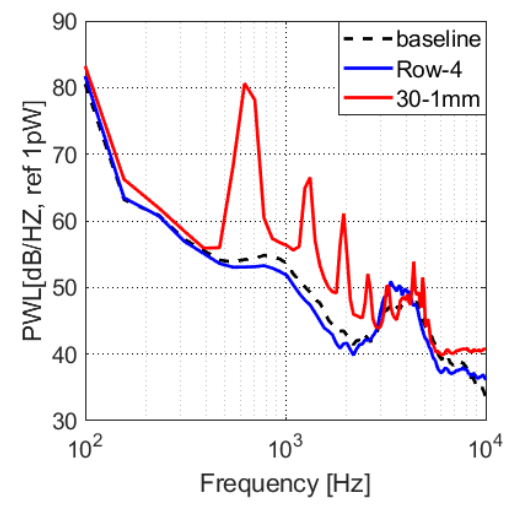

(d) $U_{0}=50 \mathrm{~m} / \mathrm{s}$

Fig. 15 the $P W L$ with different length of the porous trailing edge of untripped airfoil

\section{Conclusions}

An experimental aeroacoustic study of a NACA 65(12)-10 airfoil with solid and porous trailing edge inserts (effects of hole diameters and porous coverage) is performed with varying mean flow velocity at different attack angles. Acoustic measurements have also been conducted at $0^{\circ}$ angle of attack in order to study the effect of porous trailing edge on the laminar instability tonal noise. The key findings of this paper are listed as follows:

(1) In order to investigate the noise of the trailing edge with variations of hole diameters, $d=1,2,3 \mathrm{~mm}$ is employed for the 3 inserts with the length of the porous trailing edge up to $20 \%$ of the chord length (30mm), with the porosity at $30 \%$. The study reveals that the level of the broadband noise reduction becomes larger as the diameter of the holes decreases at lower Reynolds numbers. However, the small hole diameter will produce tonal noise at high Reynolds number. For the case of hole diameter $d=1 \mathrm{~mm}$, the distance between the two holes along the spanwise is only $1.6 \mathrm{~mm}$. This seemingly small distance would resemble a two-dimensional blunt trailing edge to encourage the formation of the bluntness-induced vortex shedding.

(2) In order to investigate the noise of the porous trailing edge with variations of the length, 15 porous trailing edge structure was designed, with the hole diameter $d=1 \mathrm{~mm}$, and porosity $\sigma=30 \%$. It shows that the longer the length of the porous trailing edge, the better in reducing the broadband noise at low speeds. However, the thickness of the first row hole near leading edge increases from $t_{h}=1.54 \mathrm{~mm}$ to $t_{h}=6.89 \mathrm{~mm}$ when the rows increased from 4 to 19. As a result, the bluntness-induced tonal peak noise level increases with the thickness of blunt edge. After analysing all the data set, we find that the tone noise will not be produced when the function $t_{h} / \delta^{*}<2$ is satisfied. 
(3) At Row-4, when $h_{c}=7 \mathrm{~mm}$, the thickest blunt thickness near trailing edge is still thin at $t_{\mathrm{h}}=1.54 \mathrm{~mm}$. As a result no bluntness-induced vortex shedding is produced even at high speed. The porous trailing edge has a very good noise reduction effect when $\alpha \leq 0^{\circ}$, but almost no noise reduction when $\alpha>0^{\circ}$.

(4) The porous trailing edge can suppress the laminar instability tonal noise, especially for the case where the length of the porous trailing edge is equal to $30 \mathrm{~mm}$ at the speed $U_{0}=20 \mathrm{~m} / \mathrm{s}$. The laminar instability noise reduction effect mainly occurs between the low and middle frequency regions $(100-7000 \mathrm{~Hz})$, and the noise increases to the high frequency region $(>7000 \mathrm{~Hz})$.

\section{Declaration of conflicting interests}

The author(s) declared no potential conflicts of interest with respect to the research, authorship, and/or publication of this article.

\section{Funding}

The author(s) disclosed receipt of the following financial support for the research, authorship, and/or publication of this article: The authors acknowledge the funding support from Shandong University of Science and Technology, grant No. 0103014.

\section{Reference}

[1] Wang Y, Zhao K, Lu XY, et al. Bio-inspired aerodynamic noise control: a bibliographic review. Applied Sciences 2019; 9; 2224:1-43

[2] Sarradj E, Fritzsche C, Geyer T. Silent owl flight: bird flyover noise measurements. AIAA Journal 2011; 49 (4): 769-779

[3] Angland D, Zhang X, Molin N. Measurements of flow around a flap side edge with porous edge treatment. AIAA Journal 2009;47(7):1660-1671

[4] T. Geyer, E. Sarradj, Noise generation by porous airfoils, In: 13th AIAA/CEAS Aeroacoustics Conference, 21-23 May 2007, Rome, Italy.

[5] T. Geyer, E. Sarradj, Trailing edge noise of partially porous airfoils, In: 20th AIAA/CEAS Aeroacoustics Conference, 16-20 June 2014, Atlanta, USA.

[6] Geyer T., Sarradj E., Fritzsche C. Measurement of the noise generation at the trailing edge of porous airfoils. Experiments in Fluids 2010; 48: 291-308

[7] Herr M., Rossignol K.S., Delfs J., Mößner M., Lippitz N. Specification of porous materials for low-noise trailing-edge applications, In: 20th AIAA/CEAS Aeroacoustics Conference, 16-20 June 2014, Atlanta, USA.

[8] Carpio A R, Martínez R M, Avallone F, et al. Experimental characterization of the turbulent boundary layer over a porous trailing edge for noise abatement. Journal of Sound and Vibration 2019; 443: 537-558

[9] Hedayati R, Carpio A R, Luesutthiviboon S, et al. Role of polymeric coating on metallic foams to control the aeroacoustic noise reduction of airfoils with permeable trailing edges. Materials 2019; 12: 1087-1100

[10]Weidenfeld M., Manela A. On the attenuating effect of permeability on the low frequency sound of an airfoil. Journal of Sound and Vibration 2016; 375:275-288

[11] Divya V, Alessandro B. Numerical modeling of flow control on a symmetric aerofoil via a porous, compliant coating. Physics of Fluids 2012; 24(9): 093601-1-19

[12] Shan J X, Zhou L, Peng X, et al. Computational analysis on porosity effect on aerodynamic characteristic of supercritical airfoil. In: Fifth International Conference on Intelligent Systems Design and Engineering Applications. 15-16 June 2014, China :310-313

[13] Sarradj E, Geyer T. Symbolic regression modeling of noise generation at porous airfoils. Journal of Sound and Vibration 2014; 333: 3189-3202

[14] Taisuke N, Atsushi H, Makoto U, et al. Evaluation of wall-interference correction method using numerical analysis and porous wall model. Journal of Aircraft 2015; 52(1): 226-234

[15]Qing A, Mahdi A, Xavier L, et al. Aerodynamic and aeroacoustic performance of airfoils with morphing structures. Wind energy 2016; 19: 1325-1339

[16]Koh S R, Zhou B, Meinke M, et al. Numerical analysis of the impact of variable porosity on trailing-edge noise. Computers and Fluids 2018; 167: 66-81

[17]Koh S R, Meinke M. Numerical analysis of the impact of permeability on trailing edge noise. Journal of Sound and Vibration 2018; 421: 348-376

[18]Zhou B Y., Koh S R, Gauger N.R., et al. A discrete adjoint framework for trailing-edge noise minimization via porous material. Computers and Fluids 2018; 172: 97-108

[19] Steven A. E., Elisha R. Prediction of noise from turbulent boundary layers attached to porous Media. AIAA Journal 2019;57(6): 2457-2464 
[20]Jiang,C., Moreau,D., Yauwenas Y, et al. Control of rotor trailing edge noise using porous additively manufactured blades. In: 24th AIAA/CEAS Aeroacoustics Conference, American Institute of Aeronautics and Astronautics, 25-29 June 2018, Atlanta, Georgia, 3792.

[21] Wang C. Trailing edge perforation for interaction tonal noise reduction of a contra-rotating fan. Journal of Vibration and Acoustics 2018; 40:1-14

[22]Carpio A R, Avallone F, Ragni D, et al. 3D-printed perforated trailing edges for broadband noise abatement. In: 25th AIAA/CEAS Aeroacoustics Conference, 20-23 May, Netherlands, 1:13

[23] Sumesh CK, Jothi TJ S. Aerodynamic noise characteristics of a thin airfoil with line distribution of holes adjacent to the trailing edge. International Journal of Aeroacoustics 2019; 18: 496-516

[24]Túlio T. R., Walter A., William R. W. On secondary tones arising in trailing-edge noise at moderate Reynolds numbers. European Journal of Mechanics / B Fluids2020; 79: 54 - 66

[25] Vathylakis A., Chong TP., Paruchuri C., Joseph, PF. Sensitivity of Aerofoil Self Noise Reductions to Serration Flap Angles. In: 22nd AIAA/CEAS Aeroacoustic Conference. American Institute of Aeronautics and Astronautics, 2 - 1 June 2016, Lyon, France, 2837.

[26] Blake, W. K., Mechanics of flow-induced sound and vibration, Volume II: Complex flow-structure interactions. Academic Press, 1986. 